\title{
EDITORIAL
}

\section{Retinal Metabolism and the Choroidal Circulation}

The papers from the Cambridge Symposium on the Choroidal Circulation and Retinal Metabolism appear in this issue of EYE. From the great diversity of papers presented it is obvious that the topic is one of great interest in which notable advances are being made.

The choroidal blood flow is higher than that of any other tissue in the body and this coupled with a low extraction rate of oxygen and glucose from the choroidal blood suggests that the choroidal blood supply is greatly in excess of the metabolic requirements of the retina. The retina obtains $80 \%$ of its oxygen and $60 \%$ of its glucose from the choroid but because of its remoteness from its source of metabolites in the choroid and the presence of several barriers to molecular movement between the choroid and retina it appears that far from being in excess the chroidal blood flow is only just sufficient to meet the metabolic needs of the retina. Indeed because of the segmental nature of the blood supply in the choroid the requirements of the retina for oxygen and glucose in the watershed zones between neighbouring areas of supply may only barely be met so that these areas are particularly vulnerable to any reduction in choroidal perfusion pressure.

In spite of the high metabolic requirements of the macular retina, macular disease directly attributable to inadequate choroidal circulation is rare. Damage to the chorio-capillaris may however be important in the genesis of central serous retinopathy. Whether this damage is mediated through excess catecholamine production in type $\mathrm{A}$ personalities remains hypothetical.

There are many different techniques for the study of the choroidal circulation including the resin cast technique which can produce such beautiful pictures and the elegant use of modern computer analysis applied to the dynamic interpretation of indocyanine green angiography.

During the Symposium a great deal of discussion was concerned with the mechanisms involved in moving water and other molecules between the choroid and the retina. The importance, distribution and even molecular structure of choroidal capillary fenestrations was discussed at length. Molecular movement between choroid and retina is dependent on both active and passive forces. Among the latter, diffusion as a result of concentration gradients, hydrostatic forces and the effects of oncotic pressure are all important. More exciting is the gradual elucidation of the complex transport systems which are present in both the apical and basal cell membranes of the retinal pigment epithelium (RPE). The transportation of water is related to the net flux of ionic movement and involves sodium, chloride, potassium and bicarbonate ions. Sugars have their own receptors and transport systems which may be blocked by competitive inhibition.

The $\mathrm{pH}$ and other aspects of intra-cellular and extra-cellular environment may have profound effects on the magnitude of molecular transport and on its direction, for the system may be changed from the absorptive to the secretory mode by, for example, alterations in the concentration of $\mathrm{K}^{+}$ions adjacent to the cell membrane or by minute alterations of intracellular $\mathrm{pH}$. As hydrogen ion production, and therefore $\mathrm{pH}$, is related to metabolic activity and particularly oxygen utilisation, and as oxidative glycolysis in the photoreceptors is greater in the. dark than in the light changes in retinal illumination have a profound effect on ionic transportation by the RPE. Changes in illumination also mediate alterations in RPE transport systems via their effect on $\mathrm{K}^{+}$ion concentrations for the concentration of $\mathrm{K}^{+}$rises in the dark and falls in the light. There are differences too in the effects of continuous as against intermittent illumination, continuous illumination reducing metabolism in the outer retina while intermittent illumination appears to increase metabolism in the inner retina.

Diamox appears to have the effect of aiding the outward movement of water to the choroid possibly as a result of acidification of the RPE and this action may be of value clinically in cases of chronic macular oedema in such conditions as retinitis pigmentosa.

The uveal effusion syndrome illustrates well the difficulties we have in understanding the 
complexities of water movement across the posterior blood ocular barrier. In the uveal effusion syndrome choroidal venous stasis or even thrombosis seems an important factor in genesis while accumulation of glycomaminoglycans in the sclera may not only increase venous stasis by compression of the vortex veins but, additionally, block an alternative outward pathway for the egress of water from the eye through the sclera.

Molecular exchange between the choroid and retina has to take place across Bruch's membrane, the RPE and the inter-photoreceptor matrix. In health Bruch's membrane poses little barrier to molecular movement but the accumulation within the membrane of lipid containing waste products of outer segment phagocytosis which is a universal concomitant of ageing, may not only alter the permeability of this membrane to molecular exchange between choroid and retina but additionally may excite a macrophagic response which in turn may have a role in the development of age-related subretinal neovascularisation. Additionally cellular immune attack on the integrity of the posterior blood ocular barrier seems to be an important factor in the pathogenesis of many types of intraocular inflammation with an immune basis.

The inter-photoreceptor matrix which lies between the RPE and the outer retina is not a homogenous layer but has structural adaptations and sheaths around cone inner and outer segments. Somewhat similar structural adaptations of the matrix probably also exist around the rods. The cone sheaths may have a role in maintaining retinal aposition and additionally may have other as yet incompletely investigated metabolic roles.

Retinoids needed for synthesis of visual pigment are transported across the RPE and through the inter-photoreceptor matrix by a series of retinoid binding proteins at least two of which reside in the RPE and an additional and important one in the matrix itself.

Lastly, it is of interest that in neoplasia many of the changes seen on angiography reflect alterations in the structure and function of the RPE rather than changes in the underlying tumour vasculature.

The interactions of the choroid and retina across the various structures which separate them are of crucial importance in many degenerative and inflammatory sight-threatening disorders of the posterior segment of the eye. The many diverse papers presented at the Cambridge Symposium highlight not only the importance of this area but gives some insight into how recent basic work may be of clinical relevance. 